\title{
Developing an Expertise Interaction Meta- Model for Group Decision Support System (GDSS)
}

\author{
Christina Albert Rayed \\ Computer and Information System dept., Sadat Academy for Management Science, Cairo, Egypt \\ *Corresponding Author:Christinalbert2004@yahoo.com
}

Copyright (C) 2013 Horizon Research Publishing All rights reserved.

\begin{abstract}
Group Decision Support System (GDSS) provides a group electronic environment in which managers and teams can collectively make decisions and design solutions for unstructured and semi-structured problems. In this paper, we propose to model for group decision support system based on expertise interaction meta-model. In this paper we develop a specialized systems such as Management Information System (MIS), Decision Support Systems (DSS), and Executive Information Systems (EIS) to be talked about work with these systems and technologies for data mining and Online Analytical Processing (OLAP) and the role of knowledge-based DSS should be to allow experts to broaden and expand their expertise, not to narrow it down to focus on the specific decision needs of managers and employees. Expert System (ES) with knowledge base captured from numerous experts in the same subject area as well as from a variety of specialists in international financial management, international accounting, international tax areas, and so forth.
\end{abstract}

Keywords Group Decision Support System (GDSS), Decision Support Systems (DSS), Interaction Meta-model, Expert System (ES), Artificial Intelligent (AI)

\section{Introduction}

Decision Support System (DSS) is an interactive computer-based system or subsystem intended to help decision makers use communication technology, data, documents, knowledge and/or models to identify and solve problems, complete decision process tasks, and make decisions. Decision support system is the area of the information systems (IS) discipline that is focused on supporting and improving managerial decision-making. Essentially, DSS is about developing and deploying IT based systems to support decision processes. A decision support system can be defined as a computer system that assists decision-makers in choosing between alternative beliefs or actions by applying knowledge about the decision domain to arrive at recommendations for the various options [1]. Many types of computer-based information systems (IS) have been developed to support decision making, including decision support systems (DSS), group support systems (GSS) and executive information systems (EIS). In an increasingly global business environment, managers of enterprises in developing countries need to utilize information technology to achieve long-run economic growth [2]. Many researches have focused attention on the capabilities of DSS system GDSS [3].

Integrated in DSS in general and GDSS in particular, they offer the potential to automate a far wider part of the overall problem-solving task than was possible with classical DSS or Expert System DSS [4].

A GDSS is composed of a set of highly configurable "tools" (e.g. brainstorming, voting and ranking, multi-criteria analysis etc.) that requires a high level of expertise for an effective use for complex decisions [5].

In integrating DSS and ES, two basic approaches are discernible and labeled expert support systems (ESS) and intelligent support systems (ISS). The key differences between these two systems are as follows. ESS is to replace human expertise with machine expertise, while ISS are to amplify the memory and intelligence of humans and groups [6]. Few would disagree with the notion that there are considerable benefits from integrating DSS and ES. The new integrated system (ESS or ISS) can support decision makers by harnessing the expertise of key organizational members. A bottleneck in the development of knowledge-based systems such as ESS is knowledge acquisition, which is a part of knowledge engineering - the process includes representation, validation, inference, explanation and maintenance. In this paper, we developed the Interaction Meta-Model contextual factors to be an Expertise Interaction Meta-Model by integrating expert systems, expertise groups and expertise tasks.

\section{Group Decision Support System}

The idea of Group Decision Support System (GDSS) is to support the decision makers at all the hierarchic levels in an 
organization to take the decisions efficiently and just in time. The main advantage of using a GDSS proved to be, over the years, a better understanding of the decision process through the involvement of all the decision makers in all the phases of the decisional issue from statement to solving and interpretation. Group Decision Support Systems (GDSS) can be presented as a set of software, hardware, language components and procedures that support a group of people engaged in a decision related meeting [7].

The GDSS is an interactive, communication and computer based system, utilized to facilitate the solution of unstructured problems by a set of co-operating decision-makers. It consists of most of the DSS elements, plus specific software to support group decisions. GDSS is a particular subclass of the more general class of computerized collaborative work systems. Special design, flexibility, ease of use, decision-making support, anonymous input, reduction of negative group behavior, parallel communications are typical characteristic features of GDSS[5].

Group decision support system (GDSS), a subclass of DSS, is defined as information technology-based support systems that provide decision making support to groups [8]. They refer to the systems that provide computer-based aids and communication support for decision-making meetings in organizations. The group meeting is a joint activity in which a group of people is interacted with equal or near-equal status. The activity and its outputs are intellectual in nature. Essentially, output of the meeting depends on the knowledge and judgment contributed by the participants. Differences in opinion may be settled by negotiation or arbitration.

Group Decision Support System is also referred to as a Group Support System (GSS), Collaborative Systems or an Electronic Meeting System since they shared similar foundations. However GDSS is characterized by being adapted for a group of people who collaborate to support integrated systems thinking for complex decision making.

Participants use a common computer or network to enable collaboration. A decision support system is an application that analyzes business data and presents it in a fashion that allows users to make business decisions more easily. A decision support system creates an environment where ideas and collaboration flourish in an efficient time-saving manner. With a decision support system can alleviate the constraints of group dynamics by facilitating more open group discussion with parallel anonymous input. Decisions are made with a higher degree of consensus and agreement resulting in a dramatically higher likelihood of implementation. With a decision support system you can bring people together like never before. GDSS contains most of the elements of decision support system plus software to provide effective support in group decision-making settings.

Group support systems have been implemented in a number of forms. Group support systems were more commonly found in larger organizations, especially financial institutions, computer service organizations, and the government. A general architecture of a GDSS includes: a) The hardware element, which is the conference facility which includes the decision room, computers, internet access, and other means of communications

b) Software tools, which are web-based applications, e-questionnaires, e-brainstorming, idea organizers, group dictionaries, questionnaire tools, policy formation tools and

c) People, who include the decision-makers themselves and, in many cases, a trained facilitator and, possibly, the support staff [9].

\section{Benefits of GDSS}

Because of the complexity and importance of performance evaluation, decision support systems are frequently used as a tool in support of decision-making. Particularly, once a decision on investment has been made, it may generate a huge profit or lead to terrible loss. Therefore, decision makers must be careful to insight a detailed before taking decisions. Decision support systems are computer-based tools that help managerial decision-making by presenting various effective alternatives. By the 1990s, knowledge-based intelligent systems have been playing an important role in new decision support tools.

Also, GDSS supports more than one person which works on a shared task. Several people work together to come up with a set of decisions to implement a solution or strategy. Most communication-driven DSS aim to support the work of internal teams, which may include external partners as well. The most intensively utilized technologies for deploying the GDSS are web-based technologies and client-server technologies. Also, groupware, bulletin boards, audio and video conferencing are utilized for communication-driven decision support.

The design issues of group decision support systems are changing because of the Web. Researchers focused for many years on what tools to provide and how to collect and aggregate input. Group Decision Support Systems give groups several advantages over many traditional, non-automated group meetings:

More participation - Because a GDSS allows anonymity, group members may be encouraged to participate because they do not feel as vulnerable to group censure for asking what may be perceived as "foolish" questions or making unpopular comments. Similarly, the participants will not be as subject to group think or conformance pressure (the reluctance to criticize the comments of others due to politeness or fear of reprisals). In addition, each group member will have more "air time" or time to contribute ideas. In non automated meetings, people must listen to others speak and pausing to reflect can cost a turn at comment or response; a GDSS allows everyone to "speak" in parallel. In a typical meeting, group members have only few minutes to express their ideas rather than the entire meeting time. In some non-automated meetings, a few group members may exert undue influence or monopolize the group's time; a GDSS makes every participant equal, eliminating member 
status incongruities. Finally, more information will be presented to the group as more participate.

Group synergy - Other group members will be able to use an idea in a manner that the originator did not because participants have different information skills. Also, the group as a whole will be better able to catch errors in a comment than the individual who proposed the idea.

Reading a comment often gives creative stimulus to others in the group. Also, groups may be more likely to consider an idea as the group's idea rather than an individual's because ideas have been merged together.

Automated record keeping - A GDSS can record all comments generated during the meeting, and consequently, the group participants may not need to take notes. In a non-automated setting, group members have to remember comments (rather than thinking of new ones) until they have a chance to speak. Participants may also forget what has been said before. In vocal meetings, some participants may not understand what was said or they may not be able to process the information quickly enough. This automated log of the discussion supports the development of an organizational memory from meeting to meeting.

More structure - A GDSS also provides a certain amount of structure to the meeting. With this structure in place, it is more difficult to deviate from the problem-solving cycle and make incomplete or premature decisions. The group has a more concentrated discussion, and they stay focused on the issues throughout the meeting. Lower levels of non-task interactions (gossiping, for example) in such groups have been observed as compared with traditional meetings.

Other benefits - As a result of more participation, group synergy, record keeping, and structure, many groups have been able to accomplish more in significantly less time necessary for traditional, non-automated meetings.

Also, these factors have contributed to higher group satisfaction with the meeting process. Finally, the new technology has enabled larger groups to meet, resulting in more information, knowledge, and skills that are brought to bear to the task at hand.

\section{GDSS Interactive Meta-Model}

GDSS is based on the standard models or on the models defined by the users. Most GDSS models address process loss by establishing recommended size ranges for group membership and norms for using and administering the systems, rather than building the process loss recognition and management directly into the systems. Also this undesirable outcome cannot be ignored when it comes to extending theoretical models of GDSS design and implementation to real-world organizations.

While the previous studies suggest that incorporating formal models of group process theories in GDSS design is feasible, it is clear that these models introduce layers of complexity that may challenge the performance of such systems.
None of these models is rich enough to support decision identification in requirements models, and there is no genuine support for decision reuse and collaboration.

The above observations raise another question, of how can inexperienced facilitators start using GDSS, at least with a reasonable probability of success. Several authors $[10,11]$ suggested an expert system approach capable to develop facilitation skills. These expert systems would include the recognition and interpretation of patterns of activity, possible facilitator's interventions and also some indication of probabilities of success.

Differences in groups using Group Decision Support Systems (GDSS) and Distributed GDSS, can be explained through differences in contextual factors (Technology, Groups and Task), and differences in the interaction of these factors. To represent this complex interplay, a novel contingency model is developed, building on the theoretical frameworks proposed by G. DeSanctis and S.R Hiltz $[12,13]$. The contextual factors (technology, groups and tasks) are represented in the main circles of the meta-model, and the interaction factors in the overlapping zones of those circles (See Figure 1). The point of the best interaction is in the interaction factor between groups, tasks and technology (GIT Interaction Factor).

The GTT Interaction Factor is essentially dynamic because it emerges from the interaction of many variables. It includes the emergent structuring of the group interaction, given the conditions created from the context in which group processes take place and the adaptation of the group to the technology and the task [14].

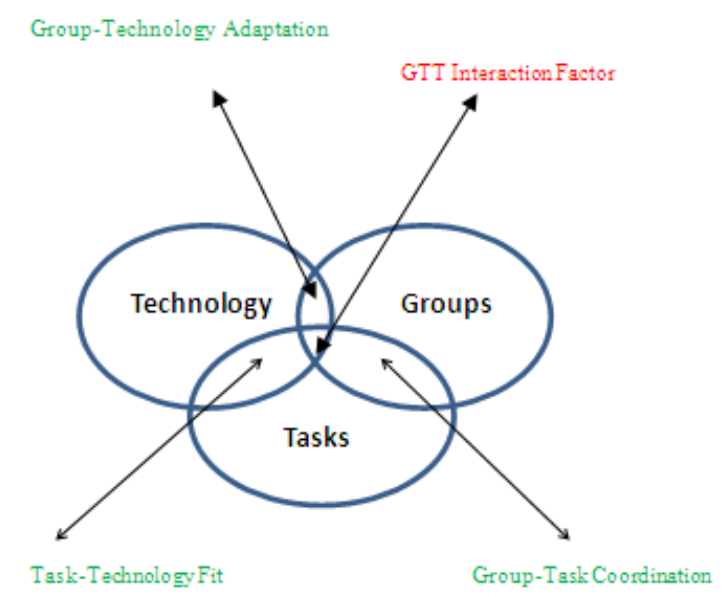

Figure 1. Interaction Meta-Model

\section{Intelligent Decision Support System}

The machine should help humans to make decision, to search for information, to control complex objects, and finally to understand the meaning of words. In order to develop intelligent computer system, we have to capture, organize and use human expert knowledge in some narrow 
areas of expertise; upgrade the computational power of the system's brain with the sophistication of algorithms using sensory processing, world modeling, behavior generation, value judgment and global communication; the amount of information and values the system has stored in its memory; and the sophistication of the process of the system functioning [15]. Besides that, intelligent system is defined as the ability of a system to act appropriately in an uncertain environment to increase the probability of success, and the success is the achievement of behavioral sub goals that support the system's ultimate goal [16].

Expert systems are often capable of supporting decision making in that domain at a level comparable to human experts. While they are flexible and often able to address complex decision problems, they are based on intuitive human reasoning and lack soundness and formal guarantees with respect to the theoretical reliability of their results. The danger of the expert system approach, increasingly appreciated by DSS builders, is that along with imitating human thinking and its efficient heuristic principles, we may also imitate its undesirable flaws.

Active DSS applications such as Expert System, Knowledge-based System, Adaptive DSS and Intelligent Decision Support System (IDSS) are categorized as part of Intelligent System studies. Intelligent systems are developed to fulfill the two main functions. Firstly, to screening, shifting and filtering the increasing overflow of data, information and knowledge. Secondly, as a supporter of an effective and productive decision making that is suitable to the user needs. Intelligent systems can be developed for these purposes; range from self-organizing maps to smart add-on modules to make the use of applications more effective and useful for the users [17].

The efficacy of Artificial Intelligent (AI) in DSS depends on AI techniques embedded in DSS. Expert System, Fuzzy Logic, Neural Network, and Genetic Algorithm are among AI techniques that have been popularized in DSS [18]. Expert System utilized human expert knowledge in its knowledge-base, capable to mimic the decision capability of human expert to assist them on their routine tasks or replace them during their unavailability [19].

Recently, there are quite a number of computer applications that have applied intelligent techniques and used DSS concepts and components. However, some researchers claim it as an essential of DSS which uses the conventional name known as IDSS and others classified it as a member of intelligent system. In this case, the application's name is given based on the intelligent techniques that they use, such as expert system which uses rules based system, knowledge based system (KBS), fuzzy sets, Neural Network for reasoning and learning capabilities. In this study, we focus our discussion on IDSS applications, which are embedded with the related intelligent techniques [20].

Intelligent Decision Support System is an integration of DSS and artificial intelligence (AI) technology combining the basic function of DSS and reasoning capabilities of AI techniques [21].

\section{Developing an Expertise Interaction Meta- Model}

Successful GDSS meetings require expertise and experience in planning meetings and building agendas. However, our experiments with current GDSS and overview of research work in the GDSS field show limited support to planning and agenda building, especially when carried out by inexperienced facilitators.

Several authors $[10,11]$ suggested an expert system approach capable to develop facilitation skills. These expert systems would include the recognition and interpretation of patterns of activity, possible facilitator's interventions and also some indication of probabilities of success.

Expert System Planner it is a prototype expert system designed to support GDSS facilitators during pre-meeting planning. The authors refer that various models of task characteristics, nature of the problem and other characteristics such as need for consensus are included in the system. Based on these models, the system makes tool recommendations to the facilitator [11]. A variety of stakeholders involved in organizational decision processes bring together their often unique viewpoints and expertise.

The progress from MIS to DSS and EIS provided models and analytical tools for professionals and usable information for executives. An expert system (ES) addresses a different type of problem: It supports the intellectual work of professionals engaged in design, diagnosis, or evaluation of complex situations that require expert knowledge in a well-defined area. The knowledge is captured not only as facts but also in the form of reasoning processes that the expert would go though in solving a problem. Some expert systems use these reasoning processes to guide a problem solving dialogue with the user.

By developing the Interaction Meta-Model contextual factors (Expert systems, Expertise groups and Expertise tasks) are represented in the main circles of the expertise meta-model, and the interaction factors in the overlapping zones of those circles as shown in Figure 2. The expert systems is including Management Information System (MIS), Decision Support System (DSS), Expert Support System, Data Mining and Online Analytical Processing (OLAP). The point of the best interaction is in the interaction factor between expertise groups, expertise tasks and expert systems is expertise groups, tasks, Systems (EGTS Interaction Factor).

The EGTS Interaction Factor is essentially dynamic because it emerges from the interaction of many expertise variables. It includes the emergent structuring of the expertise group interaction, given the conditions created from the context in which group processes take place and the adaptation of the group to the technology and the task. 


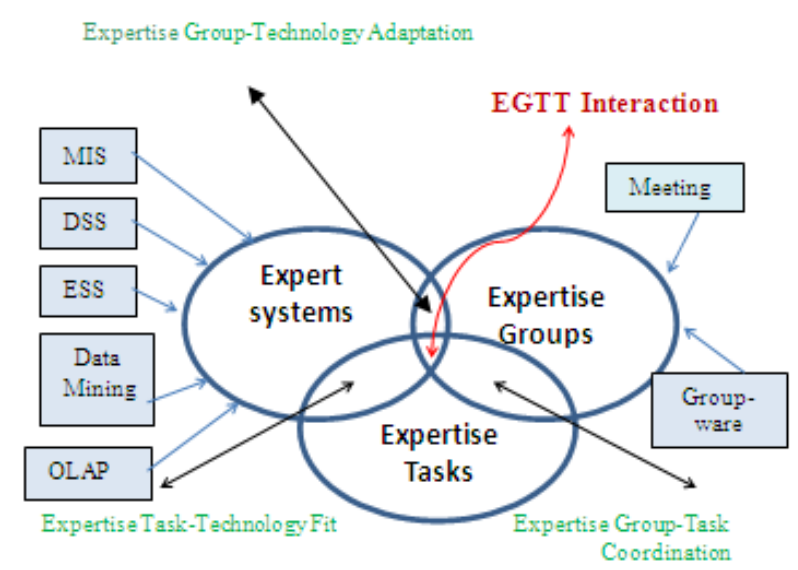

Figure 2. Interaction Meta-Model

\section{Conclusions}

There are new opportunities for enhancing decision support with data mining technologies. Some of these have led to rule oriented, automated decision systems that can be employed in real time applications. Interaction with social network systems is enabling a new level of decision support technologies in recommendation systems as well as collaborative decision systems. These tools are being used, for example, to forecast sales of cultural products such as movies and music [22, 23].

Knowledge management and the information integration technologies are essentials to manage the structured and unstructured information through whose instrumentality the good practices, the expertise and the information can be identified. As historical data is either not available or of little use in forecasting the long-term future, these probabilities are usually provided by experts. For this specific complicated problem, an expertise interaction meta-data model process from qualitative to quantitative, integrated the knowledge and intelligence of expert group, data, and useful technologies. The proposed model can organize all useful experts (or selected decision-people) to solve the task and finally form a set of problem-solving schemes, and storage into the corresponding scheme base. This modeling can be used for real life criteria, based on criteria established by the experts.

\section{REFERENCES}

[1] J.P. Shim, M. Warkentin, J.F. Courtney, D.J. Power, R. Sharda and C. Carlsson, "Past, present, and future of decision support technology", Decision Support Systems 33, 2002.

[2] Porter M., Schwab K., Lopez-Claros A. and Sala-i-Martin S., "The Global Competitiveness Report 2006-2007", World Economic Forum, Oxford Uni-versity Press, New York, NY,2006.
[3] Modarres, M., \& Beheshtian, May 2003. "Enter-prise information systems: Integrating Decision Support Systems, Executive Information Systems \& Simulation technology", ISOneWorld Confe-rence, Las Vegas, Nevada.

[4] J. Forth, K. Statis, and F. Toni, "Decision Making with a KGP Agent System", Journal of Decision-Systems, Lavoisier, 2006, vol. 15, pp. 241- 266.

[5] F. G. Filip, "Decision support and control for large-scale complex systems," Annual Reviews in Con-trol, Vol. 32, No. 1, pp. 61-70, 2008.

[6] King, D. ,'Intelligent support systems: art, augmen-tation, and agents', in R.H. Sprague, Jr and H.J. Watson (eds), Decision Support Systems: Putting Theory into Practice, 3rd edn, Englewood Cliffs, NJ: Prentice Hall, 1993.

[7] G. P. Huber, "Issues in the design of group decision support systems”, Mis Quarteley, 3(8), 1984.

[8] A. P. Sage, Decision support systems engineering, John Wiley \& Sons, New York, 1991.

[9] D. J. Power, Decision Support Systems: Concepts and Resources for Managers, Westport, Conn., Quorum Books, 2002.

[10] Niederman, F., BEISE, C., BERANEK, P., Issues and concerns about computer-supported meetings: The facilitator's perspective. Management Information Systems Quarterly, 1-22, March, 1996.

[11] AIKEN, M., SHENG, O., VOGEL, D., Integrating expert systems with group decision support sys-tems. CM Transactions on Information Systems, 9(1), 75-95, January, 1991.

[12] G. DeSanctis,. and B. Gallupe "A Foundation for the Study of Group Decision Support Systems". Management Science, $33,5,1987$.

[13] S.R Hiltz. "Distributed Group Support Systems". A GrantProposal submitted to the National Science Foundation. 1990.

[14] Fjermestad, J., Hiltz, S.R., and Turoff, M. "An In-tegrated Framework for the Study of Group Deci-sion Support Systems". Proceedings of the Hawaii International Conference on SystemSciences, Vol. IV, 1993.

[15] Negnevitsky, M., Artificial Intelligence: A guide to Intelligent Systems: Addison Wesley,England, 2005.

[16] Meystel, A. M., \& Albus, J. S., Intelligent System: Architecture, Design and Control. New York: John Wiley \& Son.Inc, 2002.

[17] Shim, J. P., Warkentin, M., Counrtney, J. F., Power, D. J., Sharda, R., \& Carlsson, C., Past, present, and future of decision support technology, Decision Support System,33(2), 2002.

[18] Wan Hussain Wan Ishak, $\mathrm{Ku}$ Ruhana Ku-Mahamud and Norita Md Morwawi, Conceptual Model of Intelligent Decision Support System Based on Naturalistic Decision Theory for Reser-voir Operation during Emergency Situation, Inter-national Journal of Civil \& Environmental Engi-neering IJCEE-IJENS Vol: 11 No: 02, 2011.

[19] V. Kildišas, Intelligent Decision Support System for 
Environmental Management". Environmental research, engineering and management, 2(16), 2001.

[20] Ger Devlin, Decision Support Systems Advances in, ISBN 978-953-307-069-8, March, 2010.

[21] F. Zhou, B. Yang, L. Li, and Z. Chen, "Overview of the New Types of Intelligent Decision Support System", International Conference on Innovative Computing Information and Control, 2008. Re-trieved from IEEE DOI: 10.1109/ICICIC.2008.
[22] Davenport, T. H., and Harris, J. G. , What People Want (and How to Predict It). MIT Sloan Man-agement Review, 2009, $50(2)$.

[23] Sharda, R., and Delen, D. , Predicting Box Office Success of Motion Pictures with Neural Networks. Expert Systems with Applications, 2006. 